\title{
Species composition and assemblage structure of microfouling diatoms growing on fiberglass plates off the coast of Yucatán, Mexico
}

\section{Composición de especies y estructura de asociaciones de diatomeas incrustantes sobre fibra de vidrio en costas de Yucatán, México}

\author{
Francisco Omar López-Fuerte ${ }^{1,2}$, David A. Siqueiros-Beltrones 3 , Lucien Velevaª and Dora A. Huerta-Quintanilla ${ }^{5}$ \\ 'Laboratorio de Sistemas Arrecifales, Depto. Académico de Economía, Universidad Autónoma de Baja California Sur. Carretera al Sur, Km. 5.5 s/n, \\ La Paz, Baja California Sur, 23080. México \\ ${ }^{2}$ Colección de Microalgas, Centro de Investigaciones Biológicas del Noroeste (CIBNOR). Avenida Instituto Politécnico Nacional 195 s/n, Col. Playa Palo de Sta. Rita, \\ La Paz, Baja California Sur, 23096, México \\ 3nnstituto Politécnico Nacional, Departamento de Plancton y Ecología Marina, Centro Interdisciplinario de Ciencias Marinas (CICIMAR). Avenida Instituto Politécnico Nacional \\ s/n, Col. Playa Palo de Santa Rita, La Paz, Baja California Sur, 23096, México \\ ${ }^{4}$ Instituto Politécnico Nacional, Laboratorio de Fisicoquímica, Departamento de Física Aplicada, Centro de Investigación y de Estudios Avanzados-Unidad Mérida (CINVESTAV) \\ Km. 6 Antigua carretera a Progreso, Cordemex, s/n, Col. Loma Bonita Xcumpich, Mérida, Yucatán, 97310, México \\ 5Instituto Politécnico Nacional, Laboratorio Nacional para el análisis de Nano y Biomateriales, CINVESTAV \\ e-mail: dsiquei@gmail.com
}

Recibido: 21 de septiembre de 2015. Aceptado: 15 de septiembre de 2016.

López-Fuerte F. 0., D. A. Siqueiros-Beltrones, L. Veleva and D. A. Huerta-Quintanilla. 2017. Species composition and assemblage structure of microfouling diatoms growing on fiberglass plates off the coast of Yucatán, Mexico. Hidrobiológica 27 (1): 23-37. DOI: 10.24275/uam/izt/dcbs/hidro/2017v27n1/Siqueiros

\begin{abstract}
Background. It is generally accepted that exopolymer films secreted by diatoms promote the onset of macrofouling on surfaces of materials used in marine environments. However, few studies provide precise information at species level regarding the microfouling process. The use of anti-fouling paints on different surfaces to create a toxic environment may aid in precluding development of the initial diatom microfilm leading to macrofouling. Goals. To describe the species composition and the structure of fouling diatoms. Methods. We analyzed assemblages growing on fiberglass plates, coated and uncoated with antifouling paint, fixed on PVC stands submerged at a 10-m depth. Because diatoms are opportunistic, fast growing microalgae that proliferate on many substrates, our hypothesis was that diatom assemblages growing on fiberglass surfaces, coated or uncoated with antifouling paint, would not differ in their structure. Results. Floristic analysis yielded 170 diatom taxa and similar assemblages with high values of diversity occurring on both surfaces. Conclusions. In keeping with our hypothesis, both colonized fiberglass surfaces compare to living substrata that favor the growth of diatom associations that exhibit high species diversity. This information will be useful in environmental studies, such as pollution abatement, and for the design and maintenance of fishing boats and industrial equipment prone to biofouling.
\end{abstract}

Key words: Bacillariophyceae, colonization, Coscinodiscophyceae, fiberglass substrate, fouling diatoms, Fragilariophyceae.

\section{RESUMEN}

Antecedentes. Las películas de exopolímeros secretadas por diatomeas promueven el establecimiento de organismos macroincrustantes en superficies de materiales utilizados en ambientes marinos. No obstante, pocos estudios proveen información taxonómica precisa a nivel especie relativa al proceso de microinscrustación. La aplicación de pinturas antiincrustantes sobre distintas superficies para crear condiciones tóxicas podrían coadyuvar en impedir el desarrollo de la micropelícula inicial de diatomeas que conlleva a la macroincrustación. Objetivos. Describir la composición de especies y la estructura de asociaciones de diatomeas incrustantes. Métodos. Se analizaron asociaciones desarrolladas sobre placas de fibra de vidrio, cubiertas con pintura antiincrustante y sin pintura, sujetas a postes de PVC sumergidos a $10 \mathrm{~m}$ de profundidad. Dado que las diatomeas son microalgas oportunistas de rápido crecimiento que proliferan sobre múltiples sustratos, nuestra hipótesis fue que las asociaciones de diatomeas que colonizarían las placas de fibra de vidrio con 0 sin recubrimiento antiincrustante no diferirían en estructura 0 composición de especies. Resultados. El análisis florístico redituó 170 taxa de diatomeas y asociaciones similares con valores altos de diversidad. Conclusiones. En acuerdo con nuestra hipótesis, ambas superficies son comparables a sustratos vivos que favorecen el crecimiento de asociaciones de diatomeas con alta riqueza de especies. Esta información será útil en estudios ambientales sobre contaminación, y en el diseño y mantenimiento de botes de pesca, así como de equipo industrial marino susceptible a colonización por microalgas.

Palabras clave: Bacillariophyceae, colonización, Coscinodiscophyceae, diatomeas incrustantes, fibra de vidrio, Fragilariophyceae, 


\section{INTRODUCTION}

Surfaces of materials used in marine environments rapidly become coated by a conditioning biofilm, also referred to as microfouling, which is highly variable in time and heterogenous in its composition (Patil \& Anil, 2008). These biofilms consist mainly of attached bacteria and diatoms, with all components enmeshed in a matrix of extracellular polymers secreted during the construction of biofilms (Cooksey et al., 1980). Microfouling modifies the substrate surface chemistry and strongly influences the subsequent colonization by macrofouling organisms. Moreover, it is generally accepted that exopolymer films secreted by diatoms promote the onset of macrofouling by conditioning the original substrate for the settlement of invertebrate larvae (Characklis \& Cooksey, 1983; Qian et al., 2003; Patil \& Anil, 2005a).

Benthic diatoms usually have been identified as major microfoulers of artificial substrata placed in the marine environment, although the source may be the water column itself or nearby surfaces that may function as islets (Fernandes et al., 1999). However, many diatom species, which turn out to be abundant in artificial substrata, are not considered typically from rocky or other hard substrata but are epipelic forms (Siqueiros-Beltrones, 2002).

The structure and composition of the microfouling community exhibits wide temporal and regional variations that are also influenced by the substratum (Cooksey et al., 1984; Patil \& Anil, 2005b). These changes in community structure are influenced by various biotic and abiotic factors and play an important role in the temporal dynamics of microfouling and macrofouling. Eventually, any diatom assemblage (regardless of the substratum) may reach a climax; then the film may degenerate and clumps of the more abundant taxa detach and drift away, becoming potential colonizers of any available surface, thus disrupting the classical colonizing sequence from pioneer (prostrated) forms to erect climax forms (Siqueiros-Beltrones, 2002). Hence, it is important to understand the species composition of diatoms during biofilm formation. Such information will be useful in environmental studies, such as pollution abatement and the design and operation of industrial equipment which are prone to biofouling.

Fouling diatoms. Extensive qualitative studies on the fouling diatom are important in order to gain understanding about autoecological processes and behavior of certain taxa under changing environmental conditions. Few studies provide precise taxonomic information at a species level in the development of microfouling processes. Of the hundreds of existing diatom genera, only a few have been documented as constant components of the microfouling, such as Amphora, Licmophora, Navicula, Nitzschia, Cocconeis, and Achnanthes. The most commonly reported species include Halamphora coffeiformis (C. Agardh) Levkov, Achnanthes longipes C. Agardh, Craspedostauros australis E. J. Cox, Toxarium undulatum J. W. Bailey, and Navicula perminuta Grunow (Molino \& Wetherbee, 2008).

Studies reveal that diatoms have specific preferences among artificial substrata (Mitbavkar \& Anil, 2000), e.g., higher diatom recruitment has been observed on fiberglass (hydrophobic) than on glass (hydrophilic) surfaces (Patil \& Anil, 2005a), although heavy fouling was incidentally documented on silicon treads used to fix aluminum foil collectors (Siqueiros-Beltrones, 2002).

Glass fiber reinforced polymer (GFRP) is one of the structural composite materials widely used in engineering applications in seawater for building and repairing boats, offshore structures in the oil industry, and many others. It is composed of two phases: the plastic one is termed the matrix, which is continuous and surrounds the fiber reinforcement embedded and dispersed into a matrix that holds it together (Loewenstein, 1973; Gupta \& Kothari, 1997).

Most of these early applications have been driven by the need to overcome the corrosion problem experienced with steel and aluminum alloys. Another reason for using GFRP has been to reduce weight, particularly the topside weight of ships. Over $95 \%$ of all composite marine craft are built with GFRP because of its low cost and excellent degradation (corrosion) resistance in seawater (Loewenstein, 1973). GFRP is usually almost free of defects, with high fiberglass content for maximum stiffness, strength, and fatigue resistance.

However, it has been recognized for several decades now that the use of anti-fouling paints on different surfaces to create a toxic environment may aid in precluding development of the initial microfilm leading to macrofouling (Robinson et al., 1985), but the effectiveness of antifouling paints may vary. Thus, because diatoms are opportunistic fastgrowing microalgae that proliferate on almost any substrate, given adequate conditions of humidity and nutrients, the concomitant hypothesis for this study posits that diatom assemblages growing on fiberglass surfaces coated with antifouling paint will not differ in their structure from assemblages growing on uncoated surfaces. To our knowledge, this is the first study on fouling diatoms growing on fiberglass surfaces, a common material used in fishing boats in the Caribbean Sea, whose duration is significantly diminished by fouling processes.

\section{MATERIALS AND METHODS}

Exposure of samples and their characterization. Biofilms grew for 2 , 4 , and 18 months on 30 (100x40x2 mm) glass-fiber-reinforced polymer plates (GFRP), with only one side coated with antifouling acrylic paint (as commercially sold for boat construction) immersed in Caribbean seawater. The polymer plates were fixed on PVC stands submerged at a $10-\mathrm{m}$ depth, $10 \mathrm{~km}$ off the Telchac marine station of CINVESTAV-Mérida, located in Yucatán, Mexico between $21^{\circ} 7^{\prime} \mathrm{N}$ and 89 $25^{\prime} \mathrm{W}$. The microfouling assays began in March 2011. After different periods of exposure, triplicate samples of each material were removed from the sea for observation to determine the type of biofouling adhered to the material surfaces. At the end of the essays in August 2012, the rest of the samples was removed for assessment. Likewise, next to the stands a sediment sample was collected with a spatula (using the top of a box of Petri as a mold); this was kept in the same Petri dish in ice and in darkness.

Seawater chemistry. Physical and chemical data were measured in the laboratory a day after each sampling. Seawater is a very aggressive medium for materials and can cause severe damage in a very short time. Usually seawater contains ions (in decreasing quantities) of $\mathrm{Cl}^{-}$, $\mathrm{Na}^{+}, \mathrm{SO}^{2-}, \mathrm{Mg}^{2+}, \mathrm{Ca}^{2+}, \mathrm{K}^{+}, \mathrm{HCO}^{3-}, \mathrm{Br}, \mathrm{B}^{3+}, \mathrm{Sr}^{2+}, \mathrm{F}^{-}$, and dissolved gases, such as $\mathrm{O}_{2}$ and $\mathrm{CO}_{2}$. Thus, seawater at $10-\mathrm{m}$ depth was analyzed for total salinity, dissolved oxygen, temperature specific sea nutrients, ammonium, silicates, phosphates, nitrites, and nitrates.

Diatom flora. Three plates were recovered after two months and processed 24 hours after being removed from the stands. Plates obtained after four and 18 months were stored for 4 weeks to separate the diatoms. The two-month microalgae film that was detached from 
both sides of the fiberglass plates (coated and uncoated) and those that were separated from the sediments were preserved in ethanol (96\%). Microalgae detached from plates submerged four and 18 months were treated as a compound sample, without differentiating coated from uncoated surfaces, and were used solely for floristic purposes. Part of the samples was observed under the microscope to ensure the existence of live diatoms.

In order to clean the diatom frustules by removing all organic matter, the samples were oxidized following the technique by SiqueirosBeltrones and Voltolina (2000) in which a mild exothermic reaction is carried out mixing a portion of the sample with commercial ethanol $(96 \%)$ and nitric acid at a 1:3:5 ratio. The oxidized material was then rinsed with tap water until a $\mathrm{pH}>6$ was reached. Afterwards, six permanent preparations were mounted using Pleurax and Zrax (IR 1.7). Cleaned material was also mounted on stubs and covered with a film of graphite spray (Aerodag $®$ G, PELCO $®$ ) for observation in a scanning electron microscope (Jeol JSM-7600F). Diatom identification was done at 400X and 1000X under a Zeiss compound microscope equipped with phase contrast, following the works of Schmidt et al. (1874-1959), Peragallo and Peragallo (1897-1908), Navarro (1982), Foged (1984), Witkowski et al. (2000), Siqueiros-Beltrones (2002), and López-Fuerte et al. (2010). The floristic list was constructed according to Round et al. (1990) and updated in www.algaebase.org (Guiry \& Guiry, 2014).

Assemblage structure. To determine the relative abundances of the diatom taxa, a sample size of 150 individuals (frustules) per slide was chosen. Numerical analyses were done twice, examining one slide from each plate and one from the sediments.

Species diversity of the diatom assemblages was estimated based on information theory $\left(\log _{2}\right)$ only on the plates submerged for two months, inasmuch as the four- and 18-month samples were not differentiated by surface (coated and uncoated), but rather as a compound sample, and thus were used for species composition analysis of the assemblages and similarity measurement by date. Values of diversity based on information theory were computed using Shannon's H' and Pielou's evenness $(J ')$. Simpson's diversity index $(1-\lambda)$ and dominance $(\lambda)$ were also estimated (Brower \& Zar, 1984) to better interpret our estimates of diversity by considering criteria that weight rare and common species differently (Siqueiros-Beltrones, 1990).

To measure similarity among diatom assemblages, samples were compared on the basis of presence/absence of species using Jaccard index, considering also their relative abundances using Bray Curtis Index (Magurran, 1988). These were fed into Program Primer V.5 based on an agglomerative classification module with flexible algorithm (Clarke \& Gorley, 2001).

\section{RESULTS}

Seawater chemistry. The seawater had total salinity of 37.48 , a pH of 7.69 , dissolved oxygen $1.1 \mathrm{ppm}$, and a temperature of $21^{\circ} \mathrm{C}$, at a depth of $10 \mathrm{~m}$. Specific nutrients were (expressed in $\mu \mathrm{M} \mathrm{L}^{-1}$ ): 1.75 ammonium, 2.61 silicates, 0.28 phosphates, 0.04 nitrites, and 1.84 nitrates.

Diatom flora. In general, the diatoms recruited on the fiberglass plates during the study were abundant and diverse (Figures 1, 2, 3). Taxonomic observations yielded 170 diatom taxa including species, varieties, and forms within 61 collected genera of diatoms from the fiberglass plates and sediments (Table 1). During the quantitative analysis, a total of 1,117 valves were counted. The Bacillariophyceae were the most diverse class with 129 taxa, the Coscinodiscophyceae yielded 22, and the Fragilariophyceae 19. As in other similar studies, diatom assemblages were dominated by several pennate species (Cassé \& Swain, 2006; Molino et al., 2009; Zargiel et al., 2011; Sweat \& Johnson, 2013). Out of the 60 identified genera, the one with most species were Amphora and Nitzschia with 13 and Diploneis and Mastogloia with 11. Meanwhile, 35 genera were represented by a single species, mostly uncommon. Sixtynine taxa were recorded only once during the quantitative phase, while 35 taxa were recorded only from the two-month submerged plates, nine from the four-month plates, four from the 18-month submerged plates, and 28 were recorded exclusively from sediments. Thus $83 \%$ of the recorded taxa in the sediments were present in the fiberglass plates. Only four taxa occurred in the three periods: Amphora turgida Gregory, Mastogloia crucicula (Grunow) Cleve, Rhopalodia musculus (Kützing) 0. Müller, and Grammatophora serpentina (Ralfs) Ehrenberg.

Overall, the estimated diversity for three of the four plates submerged for the two- month period was high; these values indicate that, besides the high species richness, there was also a somewhat homogeneous distribution of individuals among species with no single taxon being clearly dominant (Table 1). This is reflected in high-mean estimates of diversity using two indices ( $H$ ' and $1-\lambda$ ). Diversity differences between the values of the coated and uncoated side (Table 2) were minimal (higher for the assemblage from the coated side). However, said differences do not support rejection of the proposed hypothesis, i.e., structure is similar and thus diatom assemblages develop likewise on both surfaces.

Conspicuous diatom taxa, such as Cocconeis thalassiana Romero et López-Fuerte, Delphineis surirella (Ehrenberg) G. W. Andrews, Mastogloia corsicana Grunow, M. crucicula (Grunow) Cleve, and Rhopalodia musculus (Kützing) 0. Müller showed a noticeable affinity to the fiberglass plates, either coated or uncoated, both in terms of frequency and abundance. Similarity measurements using Jaccard's index segregated three associations of diatoms on the fiberglass plates (Fig. 4). However, the samples from the 4- and 18-month plates showed only a $31 \%$ similarity because they had only five species in common, suggesting two distinct assemblages. In contrast, between diatom assemblages from plates submerged 2 months and from the sediments, there was $69 \%$ similarity, since they share 89 species and were clearly segregated from the other two (Fig. 4).

Based on quantitative data, however, the Bray Curtis similarity index shows no group segregation, either between plates or according to the side of the plate (coated and uncoated). In this case, the similarity values between the three plates were very high (>95\%), even between coated and uncoated surfaces of the number two and three plates (Fig. 5).

On the basis of qualitative similarity (Jaccard's index), the apparent group formation could be deceiving (Fig. 6). However, because this type of similarity measurement tends to yield much lower values than the qualitative approach, in our experience said differences do not evidence distinct groups; accordingly, the values are relatively high and segregation is due to heterogeneous distribution of taxa. Therefore, the overall similarity analysis indicates that no effect exists due to the use of antifouling paint in terms of deterring taxa typical of a diatom film taxocoenosis, either qualitatively or quantitatively, thus supporting the $\mathrm{Ho}$. 
Table 1. Overall floristic list of diatoms observed growing on submerged fiberglass plates during 2 months (FG2), 4 months (FG4), and 18 months (FG18), and in sediments (SED), $10 \mathrm{~km}$ off the coast of Telchac, Yucatán, Mexico. Recorded only from sediments (A).

\begin{tabular}{|c|c|c|c|c|}
\hline Taxon & FG 2 & FG 4 & FG 18 & SED \\
\hline \multicolumn{5}{|l|}{ Bacillariophyceae Haeckel } \\
\hline \multicolumn{5}{|l|}{ Coscinodiscophycidae Round et R. M. Crawford } \\
\hline \multicolumn{5}{|l|}{ Coscinodiscales Round et R. M. Crawford } \\
\hline \multicolumn{5}{|l|}{ Coscinodiscaceae Kützing } \\
\hline \multicolumn{5}{|l|}{ Coscinodiscus Ehrenberg } \\
\hline 1. Coscinodiscus nitidus W. Gregory & + & & & + \\
\hline 2. C. oculus-iridis (Ehrenberg) Ehrenberg & + & & & + \\
\hline \multicolumn{5}{|l|}{ Heliopeltaceae H. L. Smith } \\
\hline \multicolumn{5}{|l|}{ Actinoptychus Ehrenberg } \\
\hline 3. Actinoptychus senarius (Ehrenberg) Ehrenberg & + & & & + \\
\hline \multicolumn{5}{|l|}{ Lithodesmiales Round et R. M. Crawford } \\
\hline \multicolumn{5}{|l|}{ Lithodesmiaceae Round } \\
\hline \multicolumn{5}{|l|}{ Tropidoneis Cleve } \\
\hline 4. Tropidoneis pusilla (Gregory) Cleve & + & & & \\
\hline \multicolumn{5}{|l|}{ Melosirophycidae E. J. Cox } \\
\hline \multicolumn{5}{|l|}{ Melosirales R. M. Crawford } \\
\hline \multirow{2}{*}{\multicolumn{5}{|c|}{$\begin{array}{c}\text { Hyalodiscaceae R. M. Crawford } \\
\text { Hyalodiscus Ehrenberg }\end{array}$}} \\
\hline & & & & \\
\hline 5. Hyalodiscus laevis Ehrenberg & + & & & \\
\hline \multicolumn{5}{|l|}{ Podosira Ehrenberg } \\
\hline 6. Podosira stelligera (Bailey) A. Mann & + & & + & + \\
\hline \multicolumn{5}{|l|}{ Paraliaceae R. M. Crawford } \\
\hline \multicolumn{5}{|l|}{ Paralia Heiberg } \\
\hline 7. Paralia sulcata (Ehrenberg) Cleve & + & & + & + \\
\hline 8. P. sulcata var. coronata (Ehrenberg) Andrews & + & & & + \\
\hline 9. P. fenestrata Sawai et Nagumo & + & & & \\
\hline \multicolumn{5}{|l|}{ Fragilariophyceae Round et R. M. Crawford } \\
\hline \multicolumn{5}{|l|}{ Climacospheniales Round } \\
\hline \multicolumn{5}{|l|}{ Climacospheniaceae Round } \\
\hline \multicolumn{5}{|l|}{ Climacosphenia Ehrenberg } \\
\hline 10. Climacosphenia moniligera Ehrenberg & + & & & \\
\hline \multicolumn{5}{|l|}{ Fragilariales P. C. Silva } \\
\hline \multicolumn{5}{|l|}{ Fragilariaceae Greville } \\
\hline Martyana Round & & & & \\
\hline 11. Martyana martyi (Héribaud-Joseph) Round & + & & & \\
\hline Podocystis J. W. Bailey & & & & \\
\hline 12. Podocystis adriatica (Kützing) Ralfs & & & & $\boldsymbol{\Delta}$ \\
\hline Licmophorales Round et R. M. Crawford & & & & \\
\hline Licmophoraceae Kützing & & & & \\
\hline Licmophora C. Agardh & & & & \\
\hline 13. Licmophora paradoxa (Lyngbye) C. Agardh & + & & & \\
\hline 14. L. remulus Grunow & + & & & \\
\hline Ulnariaceae E. J. Cox & & & & \\
\hline Hyalosynedra D. M. Williams et F. E. Round & & & & \\
\hline 15. Hyalosynedra laevigata (Grunow) D. M. Williams et Round & + & & + & + \\
\hline Opephora Petit & & & & \\
\hline 16. Opephora burchardtiae A. Witkowski, D. Metzeltin et H. Lange-Bertalot & & & & $\boldsymbol{\Delta}$ \\
\hline 17. 0. pacifica (Grunow) Petit & & & & $\Delta$ \\
\hline Synedra Ehrenberg & & & & \\
\hline
\end{tabular}


Table 1 (continuation).

\begin{tabular}{|c|c|c|c|c|}
\hline Taxon & FG 2 & FG 4 & FG 18 & SED \\
\hline 18. Synedra sp. & & + & + & \\
\hline \multicolumn{5}{|l|}{ Plagiogrammales E. J. Cox } \\
\hline \multicolumn{5}{|l|}{ Plagiogrammaceae De Toni } \\
\hline \multicolumn{5}{|l|}{ Dimeregramma Ralfs } \\
\hline 19. Dimeregramma minor (Gregory) Ralfs & + & & & + \\
\hline \multicolumn{5}{|l|}{ Plagiogramma Greville } \\
\hline 20. Plagiogramma pulchellum Greville & + & & & + \\
\hline 21. P. pulchellum var. pygmaeum (Greville) H. Peragallo et M. Peragallo & + & & & + \\
\hline 22. P. rhombicum Hustedt & + & & & + \\
\hline 23. P. wallichianum Greville & + & & + & + \\
\hline \multicolumn{5}{|l|}{ Rhabdonematales Round et R. M. Crawford } \\
\hline \multicolumn{5}{|l|}{ Grammatophoraceae Lobban etAshworth } \\
\hline \multicolumn{5}{|l|}{ Grammatophora Ehrenberg } \\
\hline 24. Grammatophora hamulifera Kützing & + & & & + \\
\hline 25. G. marina (Lyngbye) Kützing & + & & & \\
\hline 26. G. oceanica Ehrenberg & + & & & + \\
\hline 27. G. serpentina (Ralfs) Ehrenberg & + & + & + & + \\
\hline 28. G. undulata Ehrenberg & + & & & \\
\hline \multirow{2}{*}{\multicolumn{5}{|c|}{$\begin{array}{c}\text { Rhabdonemataceae Round et R. M. Crawford } \\
\text { Rhabdonema Kützing }\end{array}$}} \\
\hline & & & & \\
\hline 29. Rhabdonema adriaticum Kützing & + & & & + \\
\hline \multicolumn{5}{|l|}{ Rhaphoneidales Round } \\
\hline \multicolumn{5}{|l|}{ Psammodiscaceae Round et D. G. Mann } \\
\hline \multicolumn{5}{|l|}{ Psammodiscus Round et D. G. Mann } \\
\hline 30. Psammodiscus calceatus T. Watanabe, T. Nagumo et J. Tanaka & & & & $\Delta$ \\
\hline \multicolumn{5}{|l|}{ Rhaphoneidaceae Forti } \\
\hline \multicolumn{5}{|l|}{ Delphineis G. W. Andrews } \\
\hline 31. Delphineis livingstonii Prasad & + & & & \\
\hline 32. D. minutissima (Hustedt) Simonsen & & & & $\boldsymbol{\Delta}$ \\
\hline 33. D. surirella (Ehrenberg) G. W. Andrews & + & & & + \\
\hline 34. D. surirella var. australis (P. Petit) P. M. Tsarenko & + & & & + \\
\hline 35. D. surirelloides (Simonsen) G. W. Andrews & & & & $\boldsymbol{\Delta}$ \\
\hline \multicolumn{5}{|l|}{ Anaulales Round et R. M. Crawford } \\
\hline \multicolumn{5}{|l|}{ Anaulaceae (F. Schütt) Lemmerm } \\
\hline \multicolumn{5}{|l|}{ Eunotogramma J. F. Weisse } \\
\hline 36. Eunotogramma laeve Grunow & + & & & \\
\hline \multicolumn{5}{|l|}{ Biddulphiales Krieger } \\
\hline \multicolumn{5}{|l|}{ Biddulphiaceae Kützing } \\
\hline \multicolumn{5}{|l|}{ Biddulphia S. F. Gray } \\
\hline 37. Biddulphia biddulphiana (J. E. Smith) Boyer & + & & & \\
\hline 38. B. spinosa (J. W. Bailey) Boyer & + & & & \\
\hline \multicolumn{5}{|l|}{ Terpsinoë Ehrenberg } \\
\hline 39. Terpsinoë americana (Bailey) Grunow & + & & & \\
\hline \multicolumn{5}{|l|}{ Triceratiales Round et R. M. Crawford } \\
\hline Triceratiaceae (Schuett) Lemmermann & & & & \\
\hline Auliscus Ehrenberg & & & & \\
\hline 40. Auliscus sculptus (W. Smith) Brightwell & + & & & + \\
\hline Auricula Castracane & & & & \\
\hline 41. Auricula intermedia (Lewis) Cleve & + & & & + \\
\hline Odontella C. Agardh & & & & \\
\hline
\end{tabular}


Table 1 (continuation).

\begin{tabular}{|c|c|c|c|c|}
\hline Taxon & FG 2 & FG 4 & FG 18 & SED \\
\hline 42. Odontella aurita (Lyngbye) C. Agardh & + & & & \\
\hline \multicolumn{5}{|l|}{ Triceratium Ehrenberg } \\
\hline 43. Triceratium balearicum Cleve et Grunow & + & & & + \\
\hline 44. T. favus Ehrenberg & + & & & + \\
\hline 45. T. pentacrinus (Ehrenberg) Wallich & + & & & + \\
\hline 46. T. reticulum Ehrenberg & + & & & \\
\hline \multicolumn{5}{|l|}{ Cymatosirales Round et R. M. Crawford } \\
\hline \multicolumn{5}{|l|}{ Cymatosiraceae Hasle, Stosch et Syvertsen } \\
\hline \multicolumn{5}{|l|}{ Cymatosira Grunow } \\
\hline 47. Cymatosira Iorenziana Grunow & + & & & + \\
\hline \multicolumn{5}{|l|}{ Plagiogrammopsis Hasle, Stosch et Syvertsen } \\
\hline $\begin{array}{l}\text { 48. Plagiogrammopsis vanheurckii (Grunow) Hasle, von Stosch et Syvertsen } \\
\text { Thalassiosirales Glezer et Makarova }\end{array}$ & \multicolumn{3}{|c|}{ Thalassiosirales Glezer et Makarova } & \\
\hline \multicolumn{5}{|l|}{ Thalassiosiraceae M. Lebour } \\
\hline \multicolumn{5}{|l|}{ Ehrenbergiulva Witkowski, Lange-Bert. et Metzeltin } \\
\hline $\begin{array}{l}\text { 49. Ehrenbergiulva granulosa (Grunow) Witkowski, Lange-Bertalot et Metzeltin } \\
\text { Shionodiscus A. J. Alverson, S. H. Kang et E. C. Theriot }\end{array}$ & + & & & + \\
\hline $\begin{array}{l}\text { 50. Shionodiscus oestrupii (Ostenfeld) A. J. Alverson, S. H. Kang et E. C. Theriot } \\
\text { Thalassiosira Cleve }\end{array}$ & + & & & + \\
\hline \multicolumn{5}{|l|}{ Bacillariophycidae D. G. Mann } \\
\hline \multicolumn{5}{|l|}{ Achnanthales Silva } \\
\hline \multicolumn{5}{|l|}{ Achnanthaceae Kützing } \\
\hline \multicolumn{5}{|l|}{ Achnanthes Bory de Saint Vincent } \\
\hline 52. Achnanthes citronella (A. Mann) Hustedt & + & & & \\
\hline 53. A. curvirostrum J. Brun & & & & $\Delta$ \\
\hline \multicolumn{5}{|l|}{ Amphicocconeis M. De Stefano et D. Marino } \\
\hline $\begin{array}{l}\text { 54. Amphicocconeis disculoides (Hustedt) Stefano et Marino } \\
\text { Cocconeidaceae Kützing } \\
\text { Cocconeis Ehrenberg }\end{array}$ & + & & & + \\
\hline 55. Cocconeis britannica Naegeli & + & & + & + \\
\hline 56. C. discrepans A. W. F. Schmidt & + & & & + \\
\hline 57. C. guttata Hustedt et Aleem & & & & $\boldsymbol{\Delta}$ \\
\hline 58. C. hoffmannii Simonsen & & & & $\boldsymbol{\Delta}$ \\
\hline 59. C. krammeri Lange-Bertalot et Metzeltin & + & & & + \\
\hline 60. C. peltoides Hustedt & + & & & + \\
\hline 61. C. pseudomarginata Gregory & + & + & & \\
\hline 62. C. scutellum Ehrenberg & + & & & + \\
\hline 63. Cocconeis sp. & & + & & \\
\hline $\begin{array}{l}\text { 64. C. thalassiana Romero et López-Fuerte } \\
\qquad \begin{array}{r}\text { Bacillariales Hendey } \\
\text { Bacillariaceae Ehrenberg } \\
\text { Denticula Kützing }\end{array}\end{array}$ & + & & & + \\
\hline \multicolumn{5}{|l|}{ Nitzschia Hassall } \\
\hline 66. Nitzschia amabilis Suzuki & + & & & \\
\hline 67. N. angularis var. affinis (Grunow) Grunow & + & & & \\
\hline 68. N. bicapitata Cleve & + & & & + \\
\hline 69. N. cf. distans W. Gregory & + & + & & \\
\hline 70. N. dissipata (Kützing) Grunow & + & & + & + \\
\hline 71. N. longissima (Brébisson) Ralfs & & & & $\Delta$ \\
\hline
\end{tabular}


Table 1 (continuation).

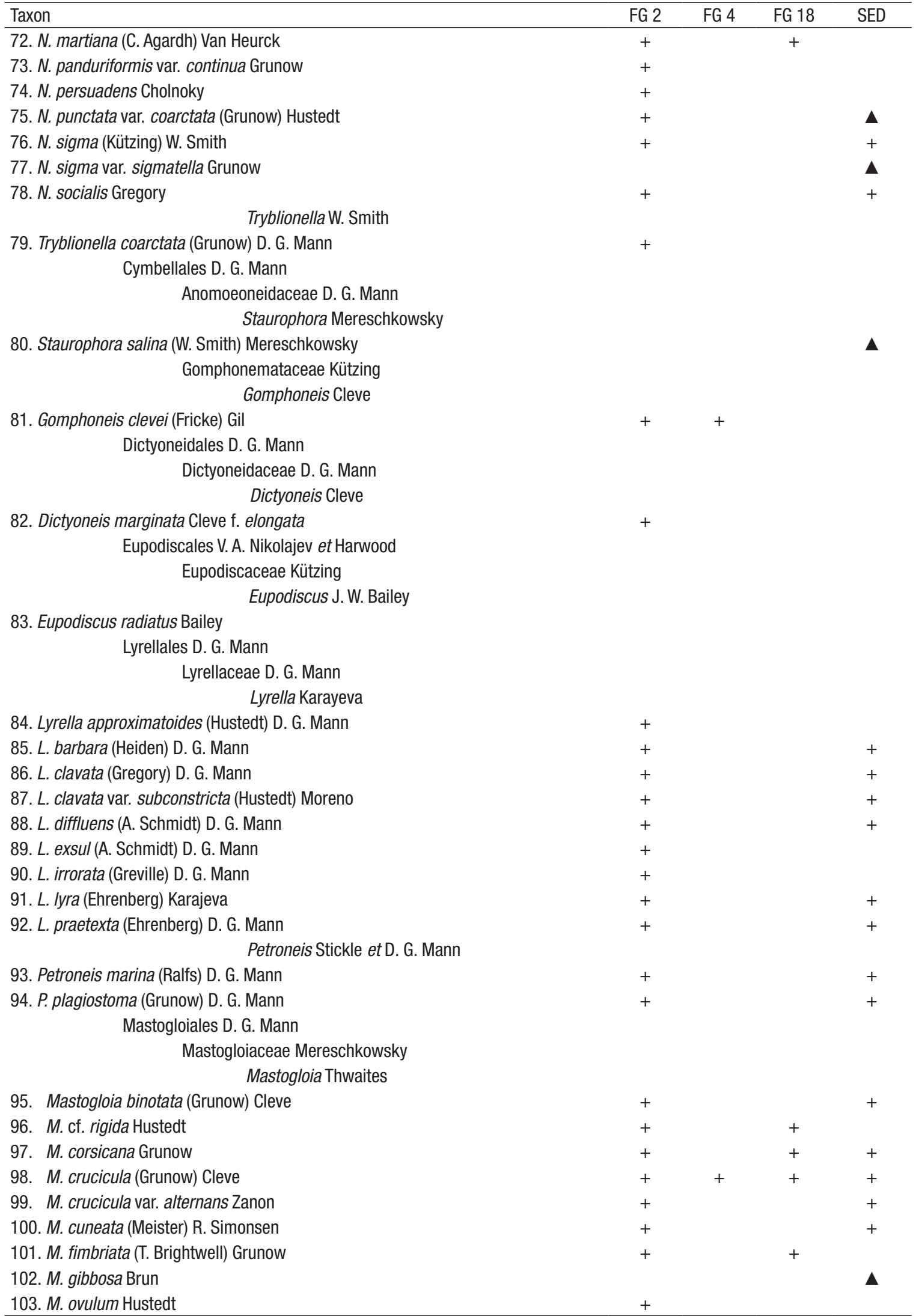


Table 1 (continuation).

\begin{tabular}{|c|c|c|c|c|}
\hline Taxon & FG 2 & FG 4 & FG 18 & SED \\
\hline 104. M. pseudolatecostata T. A. Yohn et R. A. Gibson & + & & & + \\
\hline 105. M. splendida (Gregory) Peragallo & + & & & + \\
\hline \multicolumn{5}{|l|}{ Naviculales Bessey } \\
\hline \multicolumn{5}{|l|}{ Amphipleuraceae Grunow } \\
\hline \multicolumn{5}{|l|}{ Halamphora (Cleve) Levkov } \\
\hline 106. Halamphora coffeaeformis (C. Agardh) Levkov & + & & & + \\
\hline 107. H. cymbifera (Gregory) Levkov & + & & & + \\
\hline 108. H. Iuciae (Cholnoky) Levkov & + & & & \\
\hline 109. H. turgida (Gregory) Levkov & + & + & + & + \\
\hline \multicolumn{5}{|l|}{ Diadesmidaceae D. G. Mann } \\
\hline \multicolumn{5}{|l|}{ Diploneis Ehrenberg } \\
\hline 110. Diploneis bomboides (A. W. F. Schmidt) Cleve & + & & & + \\
\hline 111. D. chersonensis (Grunow) Cleve & + & & + & \\
\hline 112. D. crabro (Ehrenberg) Ehrenberg & + & & & + \\
\hline 113. D. crabro var. dirhombus (A. Schmidt) Cleve & + & & & + \\
\hline 114. D. litoralis (Donkin) Cleve & + & & + & \\
\hline 115. D. litoralis var. clathrata (Østrup) Cleve & + & & & + \\
\hline 116. D. notabilis (Greville) Cleve & & & & $\boldsymbol{\Delta}$ \\
\hline 117. D. papula (A. W. F. Schmidt) Cleve var. papula & + & & & + \\
\hline 118. D. papula var. constricta Hustedt & + & & & \\
\hline 119. D. suborbicularis (W. Gregory) Cleve & + & & & + \\
\hline 120. D. vacillans (A. Schmidt) Cleve & + & & & + \\
\hline \multicolumn{5}{|l|}{ Caloneis Cleve } \\
\hline 121. Caloneis fossilis Cleve-Euler & + & & & + \\
\hline 122. C. liber (W. Smith) Cleve & & & & $\Delta$ \\
\hline 123. C. linearis (Grunow) Boyer & & & & $\boldsymbol{\Delta}$ \\
\hline 124. C. robusta (Grunow) Cleve & & & & $\boldsymbol{\Delta}$ \\
\hline 125. Caloneis sp. & & & + & \\
\hline \multicolumn{5}{|l|}{ Haslea Simonsen } \\
\hline 126. Haslea sp. & & & + & \\
\hline \multicolumn{5}{|l|}{ Naviculaceae Kützing } \\
\hline \multicolumn{5}{|l|}{ Navicula Bory } \\
\hline 127. Navicula (Lyrella) clavata var. distenta (Kuntz) Hustedt & + & & & + \\
\hline 128. N. arenaria Donkin & + & & & + \\
\hline 129. N. cancellata Donkin & & & & $\boldsymbol{\Delta}$ \\
\hline 130. N. clavata var. indica (Greville) Cleve & + & & & \\
\hline 131. N. digito-radiata (Gregory) Ralfs & & & & $\boldsymbol{\Delta}$ \\
\hline 132. N. directa (W. Smith) Ralfs & + & & & + \\
\hline 133. N. duerrenbergiana Hustedt & + & & & \\
\hline 134. N. Iusoria Giffen & + & & & + \\
\hline 135. N. longa (Gregory) Ralfs & + & & + & + \\
\hline \multicolumn{5}{|l|}{ Trachyneis Cleve } \\
\hline 136. Trachyneis aspera (Ehrenberg) Cleve & + & & & \\
\hline \multicolumn{5}{|l|}{ Pinnulariaceae D. G. Mann } \\
\hline \multicolumn{5}{|l|}{ Oestrupia Heiden } \\
\hline 137. Oestrupia zanardiniana (Grunow) Schrader & + & & & + \\
\hline \multicolumn{5}{|l|}{ Plagiotropidaceae D. G. Mann } \\
\hline \multicolumn{5}{|l|}{ Plagiotropis Pfitzer } \\
\hline 138. Plagiotropis delicatula (Greville) T. B. B. Paddock & & & & $\mathbf{\Delta}$ \\
\hline $\begin{array}{l}\text { 139. P. pusilla (Greville) Kuntze } \\
\text { Pleurosigmataceae Mereschowsky } \\
\text { PleurosigmaW. Smith }\end{array}$ & + & & & + \\
\hline
\end{tabular}


Table 1 (continuation).

\begin{tabular}{|c|c|c|c|c|}
\hline Taxon & FG 2 & FG 4 & $\mathrm{FG} 18$ & SED \\
\hline 140. Pleurosigma formosum W. Smith & + & & & + \\
\hline 141. P. inflatum Shadbolt & & & & $\Delta$ \\
\hline \multicolumn{5}{|l|}{ Scoliotropidaceae Mereschowsky } \\
\hline \multicolumn{5}{|l|}{ Biremis D. G. Mann et Cox } \\
\hline 142. Biremis ambigua (Cleve) D. G. Mann & + & & & + \\
\hline \multicolumn{5}{|l|}{ Sellaphoraceae Mereschowsky } \\
\hline \multicolumn{5}{|l|}{ Fallacia Stickle et D. G. Mann } \\
\hline 143. Fallacia forcipata (Greville.) Stickle et Mann & + & & & \\
\hline 144. F. nummularia (Greville) D. G. Mann & & & & $\boldsymbol{\Delta}$ \\
\hline 145. F. vittata (Cleve) D. G. Mann & & & & $\boldsymbol{\Delta}$ \\
\hline \multicolumn{5}{|l|}{ Rhopalodiales D. G. Mann } \\
\hline \multicolumn{5}{|l|}{ Rhopalodiaceae (Karsten) Topachevs'kyj et Oksiyuk } \\
\hline \multicolumn{5}{|l|}{ Epithemia Kützing } \\
\hline 146. Epithemia intermedia Fricke & + & + & & \\
\hline \multicolumn{5}{|l|}{ Rhopalodia 0. Müller } \\
\hline 147. Rhopalodia acuminata Krammer & + & & & + \\
\hline 148. $R$. gibberula var. producta (Grunow) Cleve-Euler & & + & & \\
\hline 149. R. musculus (Kützing) 0. Müller & + & + & + & + \\
\hline 150. R. pacifica Krammer & + & & & \\
\hline \multicolumn{5}{|l|}{ Surirellales D. G. Mann } \\
\hline \multicolumn{5}{|l|}{ Entomoneidaceae Reimer } \\
\hline \multicolumn{5}{|l|}{ Entomoneis Ehrenberg } \\
\hline 151. Entomoneis alata (Ehrenberg) Reimer & + & & & + \\
\hline \multicolumn{5}{|l|}{ Surirellaceae Kützing } \\
\hline \multicolumn{5}{|l|}{ Campylodiscus Ehrenberg } \\
\hline 152. Campylodiscus samoensis Grunow & + & & & \\
\hline \multicolumn{5}{|l|}{ Psammodictyon D. G. Mann } \\
\hline 153. Psammodictyon panduriforme (W. Gregory) D. G. Mann & + & & & + \\
\hline 154. P. panduriforme var. minor (W. Gregory) E. Y. Haworth et M. G. Kelly & + & & & + \\
\hline \multicolumn{5}{|l|}{ Surirella Turpin } \\
\hline 155. Surirella fastuosa (Ehrenberg) Ehrenberg & + & & & + \\
\hline 156. S. fastuosa var. cuneata 0 . Witt & + & & & + \\
\hline 157. S. fluminensis Grunow & + & & & \\
\hline 158. S. hybrida var. contracta H. Peragallo et M. Peragallo & & & & $\Delta$ \\
\hline Thalassiophysales D. G. Mann & + & & & \\
\hline \multicolumn{5}{|l|}{ Catenulaceae Mereschowsky } \\
\hline \multicolumn{5}{|l|}{ Amphora Ehrenberg } \\
\hline 159. Amphora abludens R. Simonsen & + & & & + \\
\hline 160. A. arenicola Grunow & & & & $\Delta$ \\
\hline 161. A. bigibba var. interrupta (Grunow) Cleve & & & & $\boldsymbol{\Delta}$ \\
\hline 162. A. cingulata Cleve & + & & & + \\
\hline 163. A. graeffeana Hendey & & & & $\Delta$ \\
\hline 164. A. immarginata Nagumo & & & & $\boldsymbol{\Delta}$ \\
\hline 165. A. kolbei Aleem & + & & & \\
\hline 166. A. marina W. Smith & + & & & + \\
\hline 167. A. ocellata Donkin & + & & & \\
\hline 168. A. ostrearia var. vitrea Cleve & + & & & \\
\hline 169. A. proteus Gregory & + & & + & + \\
\hline 170. A. proteus var. contigua Cleve & + & & & + \\
\hline
\end{tabular}


Table 2. Diversity values describing the structure of the diatom assemblages found growing on fiberglass plates (FG PLATES) off the coast of Yucatán, Mexico. $\mathrm{C}$ = coated, NC = Uncoated. S = Species richness; $H^{\prime}$ = Shannon's species diversity; J' = Equitatibility; $1-\lambda=$ Simpson's diversity; $\lambda=$ Dominance.

\begin{tabular}{ccccccc}
\hline FG PLATES & $\mathrm{S}$ & $\mathrm{N}$ & $\mathrm{J}$ & $\mathrm{H}^{\prime}$ & $\lambda$ & $1-\lambda$ \\
\hline 1C & 54 & 153 & 0.85 & 4.90 & 0.05 & 0.95 \\
1NC & 45 & 151 & 0.81 & 4.42 & 0.08 & 0.92 \\
2C & 44 & 159 & 0.84 & 4.60 & 0.06 & 0.94 \\
2NC & 38 & 152 & 0.81 & 4.27 & 0.09 & 0.91 \\
3C & 41 & 154 & 0.82 & 4.38 & 0.08 & 0.92 \\
3NC & 31 & 151 & 0.80 & 3.96 & 0.10 & 0.90 \\
\hline
\end{tabular}
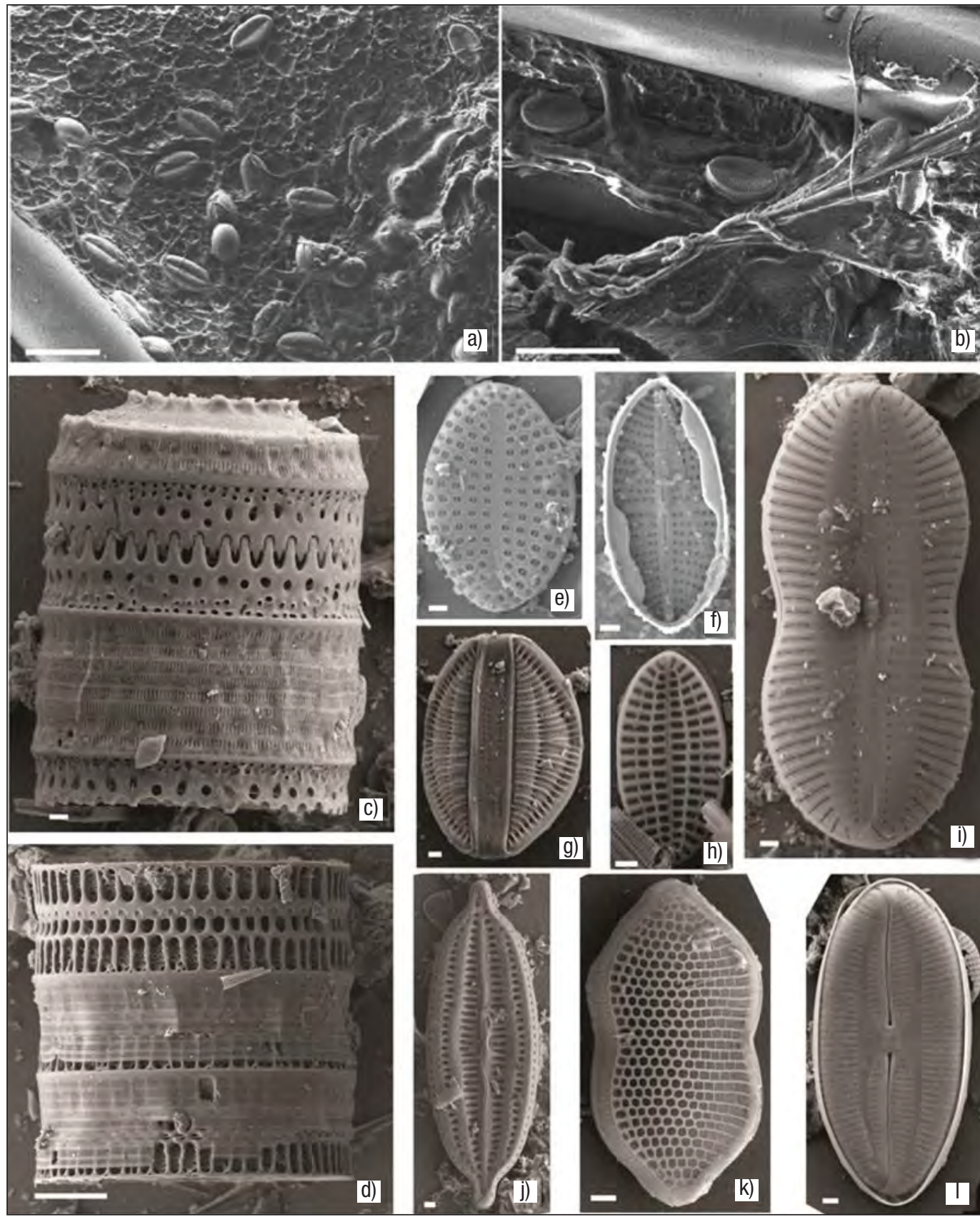

Figures 1a-l. a-b) Cocconeis sp. c) Paralia sulcata. d) Paralia fenestrata. e) Delphineis surirella. f) Mastogloia crucicula var. alternans. g) Rhopalodia musculus. h) Cocconeis discrepans. i) Diploneis bomboides. j) Mastogloia corsicana. k) Psammodictyon panduriforme var. minor. l) Fallacia forcipata. Escala: Figs. a-b, $\mathrm{d}=10 \mathrm{\mu m}$; Figs. $\mathrm{C}, \mathrm{e}-\mathrm{I}=1 \mu \mathrm{m}$. 

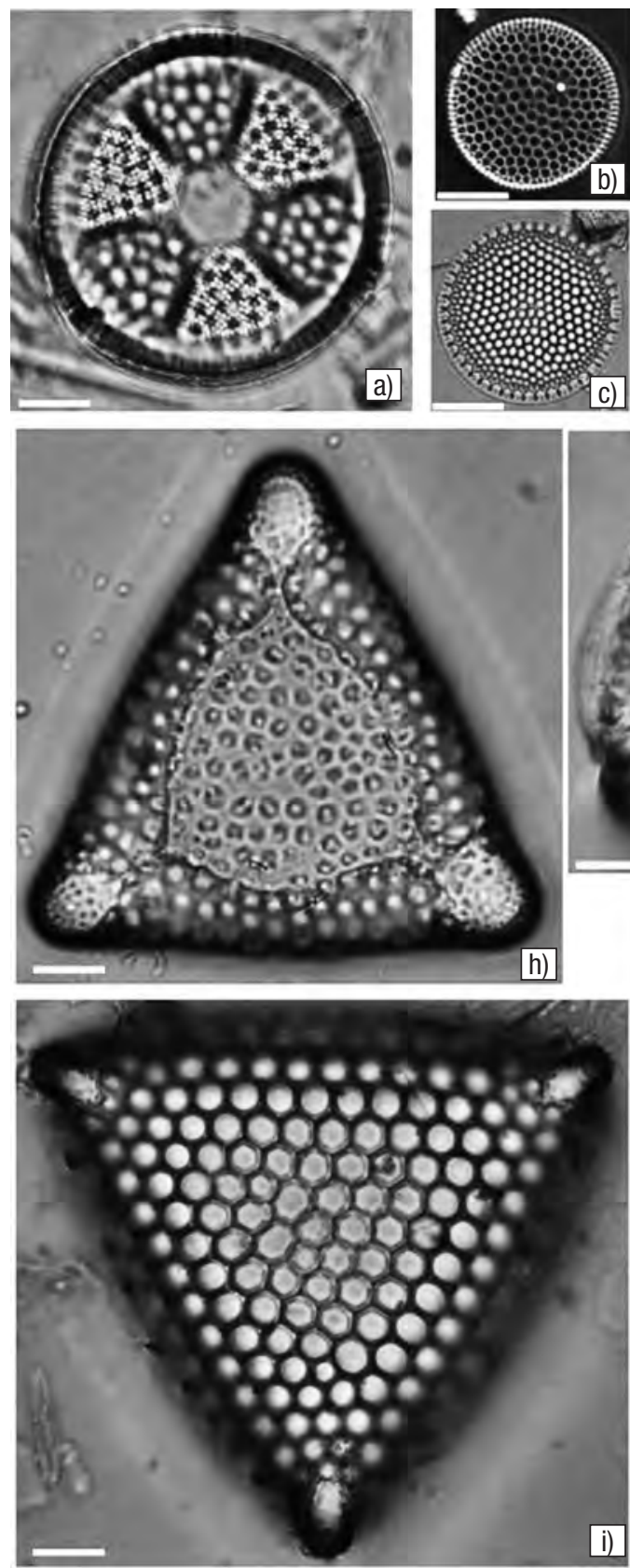
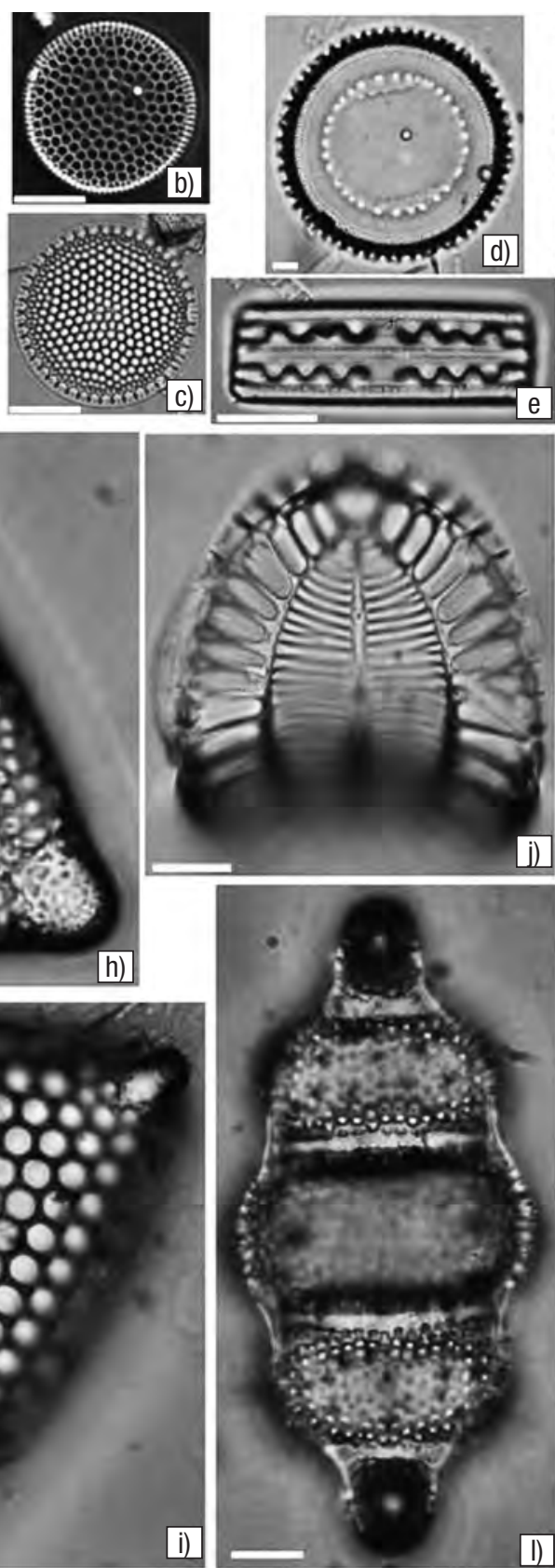
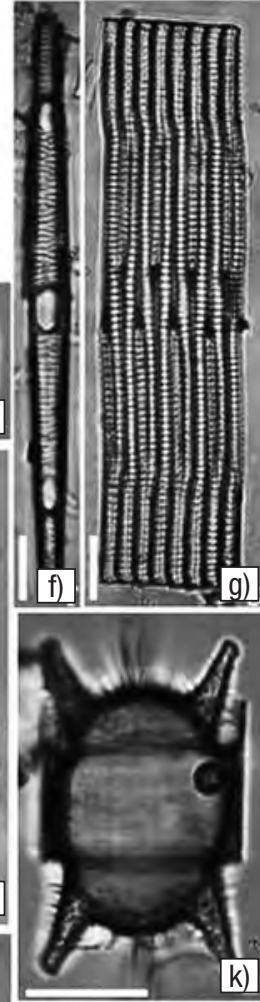

k)

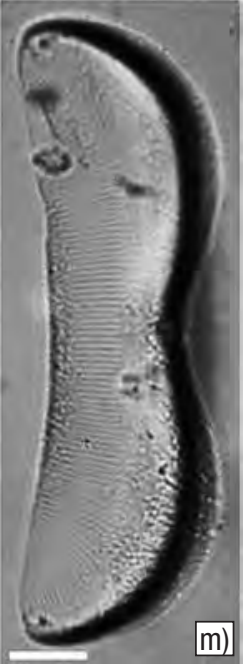

Figures 2a-m. a) Actinoptychus senarius. b) Coscinodiscus nitidus. c) Shionodiscus oestrupii. d) Paralia sulcata var. coronata. e) Grammatophora serpentina. f-g) Rhabdonema adriaticum. h) Triceratium reticulum. i) T. favus. j) Campylodiscus samoensis. k) Odontella aurita. I) Terpsinoë americana. m) Auricula intermedia. Escala $=10 \mu \mathrm{m}$.

\section{DISCUSSION}

Our results reveal that the fouling populations were heterogenous and included epipsammic, epiphytic, and epipelic (tychoplankton) species with different affinities (marine, brackish, and even freshwater). The species composition and structure (diversity, dominance, and equitability) of the diatom assemblage growing on the surface coated with antifouling paint was very similar to that of the uncoated surface, regardless of the time the plate had been submerged. Even though the computed diversity values were high, they do fall within the range-estimated values for this type of taxocoenosis (Siqueiros-Beltrones, 2005). They do, however, suggest that the colonized substrata (coated and uncoated) may be compared to those that favor the growth of diatoms such as macroalgae and seagrasses, which harbor abundant epiphytic forms. Although the number of taxa per plate appeared to be the main component of the high diversity values estimated, the distribution of individuals among the taxa was also uniform, deriving in high values of equitability. 
Many of the species found in this study belong to genera from estuarine environments that occur commonly as biofouling (Molino \& Wetherbee, 2008), including Amphora, Nitzschia, Diploneis, Mastogloia, and Navicula. As in similar studies (Characklis \& Cooksey, 1983; Cooksey et al., 1984), the assemblage in the biofilm was dominated by pennate diatoms, irrespective of the nature of the substratum and the exposure period. Anil et al. (2006) observed that pennate diatoms such as Amphora, Navicula, and Nitzschia species often dominate the fouling assemblage as well as epibiotic populations, since they are able to attach to substrates. Our observations partly agree with Amphora, Nitzschia, Mastogloia, and Diploneis being the dominant taxa; the latter two taxa are very common in sediments.
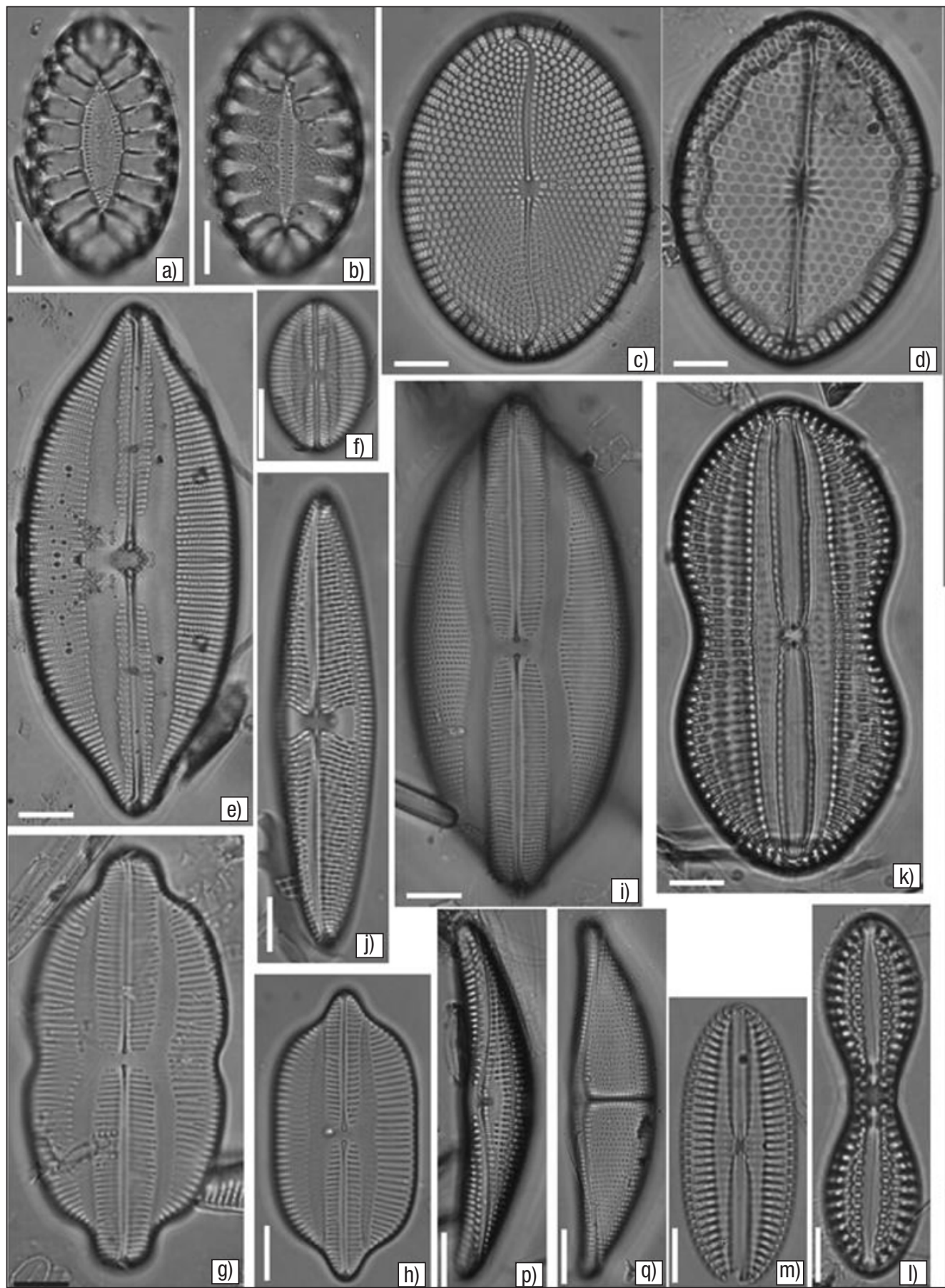

)
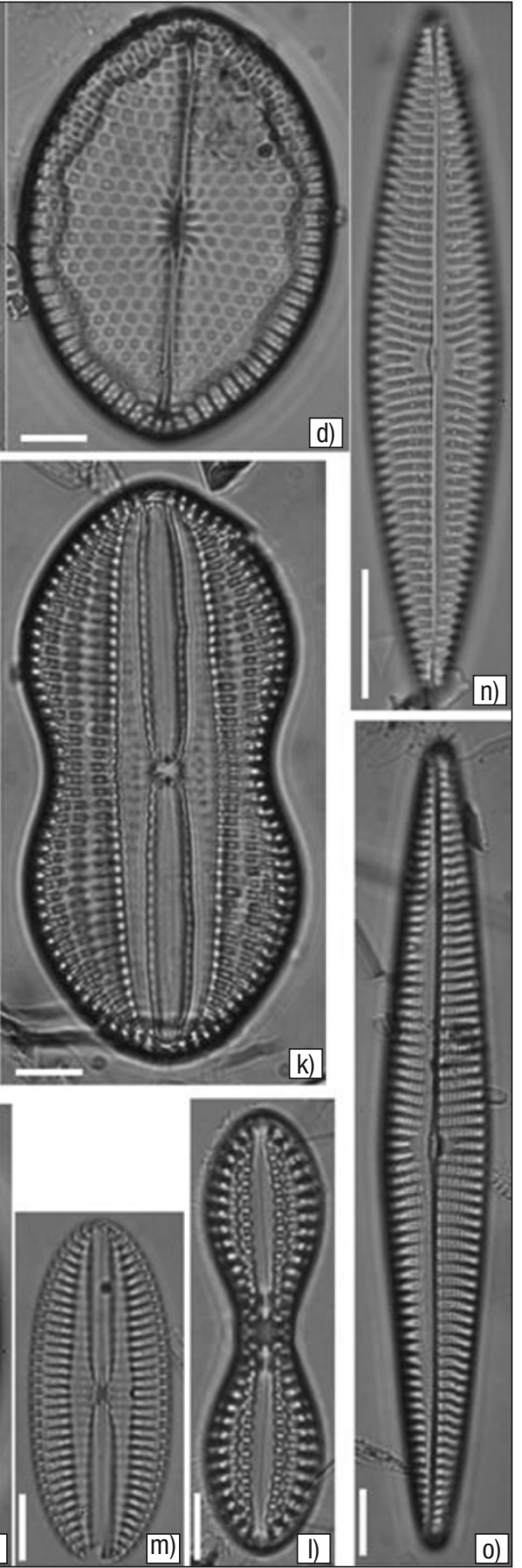

Figures 3a-q. a) Surirella fastuosa. b) S. fastuosa var. cuneata. c) Mastogloia splendida. d) M. fimbriata. e) Lyrella approximata. f) L. barbara. g) Navicula (Lyrella) clavata var. distenta. h) Navicula (Lyrella) caribaea. i) Lyrella lyra. j) Trachyneis aspera. k) Diploneis bomboides. I) D. crabro var. dirhombus. m) D. papula var. papula. n) Navicula arenaria var. arenaria. o) N. Ionga. p) Amphora proteus. q) A. ostrearia var. vitrea. Escala $=10 \mu \mathrm{m}$. 
The examination of the sediment adjacent to the structure holding the fiberglass plates rendered an accurate reference of the origin of the diatoms found on the plates, because many of the diatom taxa have been recorded from sediments (83\%). These observations indicate that the dominance of certain species in the biofilm can be attributed to their dominance in the adjacent sediments, resulting in a higher substratum-encountering probability. Benthic diatoms that are frequently re-suspended by hydrodynamic or biotic processes may, after a while, colonize a different available substrate (Breznak et al., 1985). Once attached, the growth and assemblage structure is further dependent upon the physicochemical and biological nature of both the ambient water and the substrate surface. Studies reveal that diatoms have specific substrate preferences (Mitbavkar \& Anil, 2000), and higher diatom recruitment has been observed on fiberglass (hydrophobic) than on a hydrophilic surface such as glass (Patil \& Anil, 2005a). Due to its physical characteristics, the fiberglass plates allow initially for the settlement of small forms $(<20 \mu \mathrm{m})$ of diatoms (Figures 1a-b), which in turn make the substrate favorable for large forms (Figures 1c-I). Also, the presence of sand on both sides of the plates may be a factor because diatoms from the sediments (epipelic and epipsammic) may migrate from the sediments (Round et al., 1990) to the available space in the plates, finding it suitable for growth and colonizing it, as observed in this experiment. Heavy diatom growth has been known to occur as thick films in certain artificial substrata such as PVC surfaces and silicon, where up to 178 taxa have been recorded for colonization periods of three weeks (Siqueiros-Beltrones, 2002).

The assemblage structure was similar to that recorded for many studies on benthic diatoms, i.e., few abundant species, many common species, and many more rare species, but once either in the qualitative or quantitative analysis. The number of identified taxa in this investigation is similar to diatom assemblages from natural substrata, e.g., López-Fuerte et al. (2013), in which 106 taxa were recorded living as epiphytes of Thalassia testudinum K. D. Koenig in Yalahau lagoon, Quintana Roo, Mexico. On the other hand, in comparison with other studies that used artificial substrata, the number of diatom taxa recorded here is much higher, e.g., Fernandes et al. (1999) and Patil \& Anil (2005b) reported 60 and 51 taxa, respectively, growing on glass slides.

The marked difference in the number of taxa recovered from the fiberglass plates from one period of submergence to another, i.e., 135 taxa in the two-month plates vs. 11 and 21 taxa for the four- and eighteen-month plates, may be explained by the fact that when diatom films reach a certain degree of growth, lumps then begin to detach from the films and so provide colonizing material for other substrata (Siqueiros-Beltrones, 2002). Thus, much of the diatom flora from the late phases of succession would be lacking in the plates submerged for longer periods.

Because exopolymer films secreted by diatoms promote the onset of macrofouling by conditioning the original substrate for invertebrate larvae, it is necessary to acquire a precise knowledge of the microfouling species from the initial phases of microfouling in order to better understand the ecological processes that may be helpful for controlling fouling events. Likewise, the development of anti-fouling paints should focus on avoiding the settlement and growth of diatoms, thus delaying the onset of macrofouling that generally depends on the modulation of the substrate by pioneer microfouling diatoms.

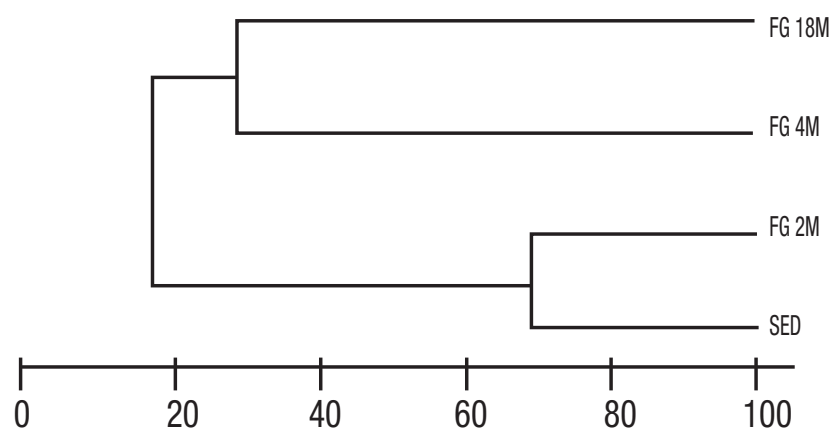

Figure 4. Similarity of diatom assemblages grown on fiberglass ( $\mathrm{FG}$ ) plates and sediments (SED), based on Jaccard's similarity index. $M=$ Months.

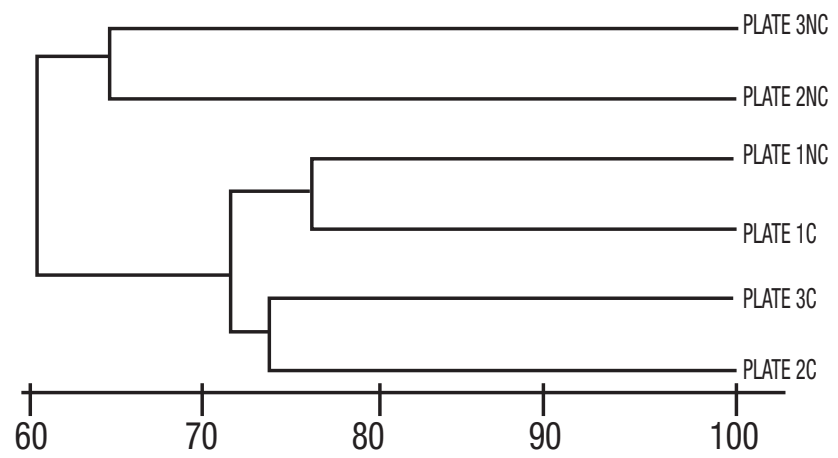

Figure 5. Similarity (Bray Curtis) of diatom assemblages grown only on fiberglass plates, with no group segregation. $\mathrm{C}=$ coated, $\mathrm{NC}=$ uncoated, surfaces.

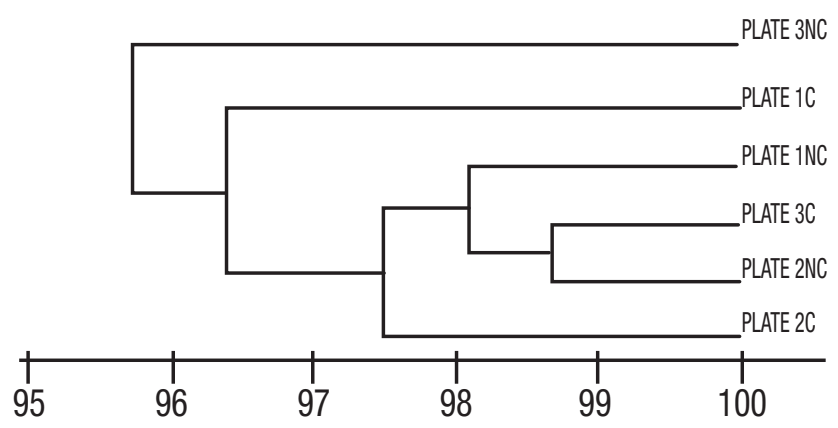

Figure 6. Similarity of diatom assemblages grown only on fiberglass plates, based on Jaccard's index with group segregation. C = coated, NC = uncoated, surfaces.

Overall, the above results suggest a greater ability of benthic diatoms to colonize fiberglass surfaces, which is probably explained by a higher degree of contact between the cells and the surface. Moreover, as observed in natural substrata, the structure of diatom assemblages showed variations that may be attributed to how long the plates were submerged. In order to sustain this, intermediate submersion times should be implemented in order to allow other successional phases to be detected. 
Because the microfouling diatom assemblages may provide suitable conditions for the onset of macrofouling, we suggest further research be undertaken in order to better understand their successional processes, which may aid in developing an efficient strategy for preventing or delaying the settlement of macrofouling organisms.

\section{ACKNOWLEDGEMENTS}

This research was supported by the Mexican Council of Science and Technology (CONACYT) under Grant 179110. SEM micrographs were obtained at the Laboratory of Microscopy, CINVESTAV, Unidad Mérida, Mexico. FOLF currently holds a postdoctoral research grant from CONACYT. DASB is COFAA and EDI fellow at the IPN. We acknowledge reviews by the appointed referees and editors.

\section{REFERENCES}

Anil, A. S., M. Mitbavkar S. D'silva, P. Hegde, S. D'costa, D. Meher \& D. BanerJEE. 2007. Effect of ageing on survival of benthic diatom propagules. Journal of Experimental Marine Biology and Ecology 343 (1): 37-43. DOI: ORG/10.1016/J.JEMBE.2006.11.006

Breznak, J. A., K. E. Cooksey, F. E. Eckardt, W. Filipz, M. Fletcher, R. J.Gibbons, H. Gude, W. A. Hamilton, T. Hattori, H. G. Hoppe, A. G. Matthysse, D. C. SavaGe \& M. SHIL0. 1985. Activity on surfaces. In: Marshall, K. C. (Ed.) Microbial Adhesion and Aggregation. Springer, Berlin. pp. 203-221.

CASSÉ, F. \& G. W. Swaln. 2006. The development of microfouling on four commercial coatings under static and dynamic immersion. International Biodeterioration \& Biodegradation 57:179-185. D0l: $10.1080 / 08927014.2011 .609616$

Characklis, W. G. \& K. E. Cooksey. 1983. Biofilm and microbial fouling. Advances in Applied Microbiology 29: 93-148.

Clarke, K. R. \& R. N. Gorley. 2001. PRIMER v5: user manual/tutorial. Primer-E. Plymouth: Plymouth Marine Laboratory.

Cooksey, B., K. E. Cooksey, C. A. Miller \& J. H. Paul. 1980. Attachment of diatoms to surfaces: field and laboratory experiments. In: Berkley, R. C. W., J. M. Lynch, J. Melling, P. R. Rutter \& Vincent E. (Eds.). Proceedings of the microbial adhesion to surfaces symposium. EllisHorwood, Chichester (UK), pp. 526-528.

Cooksey B., K. E. Cooksey, C. A. Miller, J. H. Paul, R. W. Rubin \& D. Webster. 1984. The attachment of microfouling diatoms. In: Costlow J. D. \& Tipper, R. C. (Eds.). Marine biodeterioration: an interdisciplinary study. US Naval Institute. Annapolis, MD, pp. 167-171.

Fernandes, L. F., F. P. Brandini, K. S. Gutzeit, A. L. Fonseca \& F. M. Pellizzari. 1999. Benthic diatoms growing on glass slides in the Paranagua Bay, southern Brazil: Taxonomic structure and seasonal variation. Revista do Horto Botanico 28: 53-100.

Foged N. 1984. Freshwater and littoral diatoms from Cuba. Bibliotheca Diatomologica, Band 5. J. Cramer. Vaduz. 243 p.
GuIRY, M. D. \& G. M. GuIRY. 2014. AlgaeBase. World-wide electronic publication, National University of Ireland, Galway. http://www.algaebase.org; (accessed: 19 May 2014).

Gupta, V. B. \& V. K. Kothari. 1997. Manufactured Fibre Technology. Chapman and Hall. pp. 544-546.

Loewenstein, K. L. 1973. The Manufacturing Technology of Continuous Glass Fibers. New York: Elsevier Scientific. pp. 2-94.

López-Fuerte, F. O., D. A. Siqueiros-Beltrones \& J. N. Navarro. 2010. Benthic diatoms associated with mangrove environments in the Northwest region of México. CONABI0-UABCS-CICIMAR-IPN. 206 p.

MagurRan, A. E. 1988. Ecological diversity and its measurement. Princeton University Press, Princeton, NJ, 192 p.

MitbavkaR, S. \& A. C. AniL. 2000. Diatom colonization on stainless steel panels in estuarine waters of Goa, west coast of India. Indian Journal of Marine Sciences 29: 273-276.

Molino, P. J. \& R. Wetherbee. 2008. The biology of biofouling diatoms and their role in the development of microbial slimes. Biofouling 24: 365-379. DOI: 10.1080/08927010802254583

Molino, P. J., E. Campbell \& R. Wetherbee. 2009. Development of the initial diatom microfouling layer on antifouling and fouling-release surfaces in temperate and tropical Australia. Biofouling 25: 685-694. DOI: 10.1080/08927010903089912

NavarRo, J. N. 1982. Marine diatoms associated with mangrove prop roots in the Indian River, Florida, USA. Bibliotheca Phycologica, Band 61. Vaduz: Lubretch \& Cramer Ltd. 151 p.

PatL, J. S. \& A. C. AnIL. 2008. Temporal variation of diatom benthic propagules in a monsoon-influenced tropical estuary. Continental Shelf Research 28 (17): 2404-2416.

PatL, J. S. \& A. C. ANIL. 2005a. Influence of diatom exopolymers and biofilms on metamorphosis in the barnacle Balanus amphitrite. Marine Ecology Progress Series 301 (23): 1-45.

PATL, J. S. \& A. C. ANIL. 2005b. Biofilm diatom community structure: influence of temporal and substratum variability. Biofouling 21: 189206. DOI: 10.1080/08927010500256757

Peragallo, H. \& M. Peragallo. (1897-1908) Diatomées marines de France et des Districts Maritimes Voisins. Grez-sur-Loing, France: M.J. Témpere. $580 \mathrm{p}$.

Qian, P. Y., V. Thiyagarajan, S. C. K. Lau \& S. C. K. Cheung. 2003. Relationship between bacterial community profile in biofilm and attachment of the acorn barnacle Balanus amphitrite. Aquatic Microbial Ecology 33: 225-37.

Robinson, M. G., B. D. Hall \& D. Voltolina. 1985. Slime films on antifouling paints; short-term indicators of long-term effectiveness. Journal of Coatings Technology 57 (725): 35-41. 
Round, F. E., R. M. Crawford \& D. G. Mann. 1990. The diatoms: biology and morphology of the genera. Cambridge University Press, New York. $747 \mathrm{p}$.

Schmidt, A, M. Schmidt, F. Fricke, H. Heiden, 0. Müller, F. Hustedt. 18741959. Atlas der diatomaceenkunde. Reisland, Leipzig: Berlin Akademie-Verlag. 460 pls.

Siqueiros-Beltrones D.A. 2002. Diatomeas bentónicas de la Península de Baja California; diversidad y potencial ecológico. La Paz, México: IPN-CICIMAR-UABCS. 246 p.

Siqueiros-Beltrones, D. A. \& D. Voltolina. 2000. Grazing selectivity of red abalone Haliotis rufescens postlarvae on benthic diatom films under culture conditions. World Aquaculture 31 (2): 239-246. D0I: 10.1111/J.1749-7345.2000.TB00359.X
SwEAT, L. H. \& K. B. JOHnson. 2013. The effects of fine-scale substratum roughness on diatom community structure in estuarine biofilms. Biofouling 29: 879-890. D0: 0RG/10.1080/08927014.2013.811492

Zargiel, K. A., J. S. Coogan \& G. W. Swain. 2011. Diatom community structure on commercially available ship hull coatings. Biofouling 27: 955-965. DOI: ORG/10.1080/08927014.2011.618268

Witkowski, A, H. Lange-Bertalot \& D. Metzeltin. 2000. Diatom flora of marine coasts I. In: Lange-Bertalot, H. (Ed.). Annotated diatom micrographs. Königstein, Germany: A. R. G. Gantner Verlag K. G., Iconographia Diatomologica 7. Diversity-Taxonomy-Identification. Koeltz Scientific Books. 925 p. 